\title{
CORE EQUIVALENCE IN ECONOMY UNDER AWARENESS
}

\author{
TAKASHI MATSUHISA \\ Department of Natural Sciences, Ibaraki National College of Technology \\ Nakane 866, Hitachinaka-shi, Ibaraki 312-8508, Japan \\ E-mail: mathisa@ge.ibaraki-ct.ac.jp
}

\begin{abstract}
The extended notion of pure exchange economy under uncertainty, called an economy with awareness structure, is presented, where each trader having strictly monotone preferences makes decision under his/her awareness and belief. We show an extension of the core equivalence theorem: The ex-post core coincides with the set of all generalized expectations equilibria in awareness for the economy.
\end{abstract}

1. Introduction. This article relates economies and distributed belief. The purposes are: First, to present an extended notion of economy under uncertainty, called an economy with awareness structure, where each trader makes decision in his/her awareness and belief under incomplete information. Secondly, to show the 'core equivalence theorem' under generalized expectations equilibrium in the extended economy:

MAIn TheOREm. In a pure exchange economy under uncertainty, the traders are assumed to have an awareness structure and and they are risk averse. Then the ex-post core coincides with the set of all expectations equilibria in awareness for the economy.

Recently researchers in such fields as economics, AI, and computer science have become interested in reasoning of belief and knowledge. There are pragmatic concerns about the relationship between knowledge (belief) and actions. Of most interest to us is the emphasis on situations involving the distributed knowledge (belief) of a group of agents rather than that of a single agent. At the heart of any analysis of such situations as a conversation, a bargaining session or a protocol run by processes is the interaction between

2000 Mathematics Subject Classification: Primary 91B50, 91B60; Secondary 03B45.

Journal of Economic Literature Classification: D51, C78, D61.

Key words and phrases: awareness, belief, core equivalence, expectations equilibrium in awareness, ex-post core, Pareto optimum, pure exchange economy.

The research is partially supported by the Grant-in-Aid for Scientific Research (C)(2) (No. 14540145) in the Japan Society for the Promotion of Sciences.

The paper is in final form and no version of it will be published elsewhere. 
agents. An agent in a group must take into account not only events that have occurred in the world but also the knowledge of the other agents in the group.

Many authors have investigated several notions of core in an economy under asymmetric information (e.g., Wilson [14], Volij [13], Einy et al [6] and Forges et al [9]). The serious limitations of their analysis is the assumption of the 'partition' structure of information the traders receive, together with the assumption of common-knowledge on traders' willingness to trade. From the epistemic point of view, the partition structure represents the traders' knowledge satisfying the postulates: 'Truth' $\mathbf{T}$ (what is known is true), the 'positive introspection' 4 (that we know what we do) and the 'negative introspection' $\mathbf{5}$ (that we know what we do not know). The postulate $\mathbf{5}$ is indeed so strong that it describes the hyper-rationality of traders, and thus it is particularly objectionable. So is the common knowledge assumption because the notion of common knowledge is defined by an infinite recursion of all of individual knowledge.

This article explores the extent to which two assumptions, partition structure and common-knowledge, are gneralized in economies under uncertainty. As has already been pointed out in Geanakoplos [8], this relaxation can potentially yield important results in a world with imperfectly Bayesian agents.

The idea has been performed in different settings. Among other things Geanakoplos [8] showed the no speculation theorem in the extended rational expectations equilibrium under the assumption that the information structure is reflexive, transitive and nested (Corollary 3.2 in Geanakoplos [8]). The condition 'nestedness' is interpreted as a requisite on the 'memory' of the trader. Recently, Matsuhisa, Ishikawa and Hoshino [11] extended the core equivalence theorem in an economy under generalized information structure without the nestedness condition.

This article is organized as follows: In Section 2 we begin with illustrating our model by a simple example. In Section 3 we propose the model: An economy with awareness structure. In Section 4 we present the notions of expectations equilibrium in awareness and ex-post core in the economy. We state explicitly the core equivalence theorem and sketch the proof. Finally we conclude by giving some remarks.

2. Illustrative example. Let us consider the exchange economy under uncertainty as follows: There are two traders 1 and 2, and two commodities $x$ and $y$. We let $x_{t}$ and $y_{t}$ respectively mean the amounts of commodities $x$ and $y$ that the trader $t$ possesses. Let $\Omega$ be the state space $\left\{\omega_{1}, \omega_{2}, \omega_{3}\right\}$. The traders' information structures are given as follows: Trader 1 has the partition information $P_{1}(\omega)=\{\omega\}$ for all $\omega \in \Omega$, but trader 2 has the non-partition information $P_{2}\left(\omega_{1}\right)=\Omega, P_{2}\left(\omega_{2}\right)=\left\{\omega_{1}, \omega_{2}\right\}$ and $P_{2}\left(\omega_{3}\right)=\left\{\omega_{1}, \omega_{3}\right\}$. Trader 1 has her initial endowment $e_{1}(\omega)=\left(\frac{3}{2}, 3\right)$ for every $\omega \in \Omega$, and trader 2 has his initial endowment $e_{2}(\omega)=(1,1)$ for every $\omega \in \Omega$. Furthermore they have the utilities $U_{1}$ and $U_{2}$ as follows: $U_{1}\left(x_{1}, y_{1} ; \omega\right)=x_{1}^{\frac{1}{3}} y_{1}^{\frac{2}{3}}$ for every state $\omega \in \Omega, U_{2}\left(x_{2}, y_{2} ; \omega_{1}\right)=U_{2}\left(x_{2}, y_{2} ; \omega_{2}\right)=$ $x_{2} y_{2}, U_{2}\left(x_{2}, y_{2} ; \omega_{3}\right)=x_{2}^{\frac{1}{2}} y_{2}^{\frac{1}{2}}$, and they have different priors: $\pi_{1}(\omega)=1 / 3$ for every state, and $\pi_{2}\left(\omega_{1}\right)=1 / 2$ and $\pi_{2}(\omega)=1 / 4$ for $\omega \neq \omega_{1}$.

In the economy, we can see that traders' initial endowments $\left(e_{1}, e_{2}\right)$ are ex-ante Pareto optimal. and we can observe that equilibrium price $p=\left(p_{1}, p_{2}\right)$ is the same between 
traders $1,2\left(p_{1}=p_{2}\right)$, and the endowments give a generalized notion of rational expectations equilibrium for the price $p$. It is noted that trader 2's information $\sigma(p) \cap P_{2}$ given by the price may be still a non-partition.

This situation is very interesting: Even though traders have different priors and they process different information, they do not trade at all. It should be noted that $P_{2}$ satisfies the reflexivity: For any $\omega \in \Omega, \omega \in P_{2}(\omega)$, however it does not satisfy the transitivity: $P_{2}(\xi) \subseteq P_{2}(\omega)$ whenever $\xi \in P_{2}(\omega)$. Moreover $P_{2}$ is not nested: An information structure $\left(P_{i}\right)_{i \in N}$ is said to be nested if for each $i \in N$ and for all states $\omega$ and $\xi$ in $\Omega$, either $P_{i}(\omega) \cap P_{i}(\xi)=\emptyset$, or else $P_{i}(\omega) \subseteq P_{i}(\xi)$ or $P_{i}(\omega) \supseteq P_{i}(\xi)$. In this article we shall investigate the pure exchange economies under generalized information structure as like this example.

3. The model. Let $\Omega$ be a non-empty finite set called a state space and $2^{\Omega}$ the field consisting of all subsets of $\Omega$. Each member of $2^{\Omega}$ is called an event and each element of $\Omega$ called a state. The space of the traders is a measure space $(T, \Sigma, \mu)$ in which $T$ is a set of traders, $\Sigma$ is a $\sigma$-field of subsets of $T$ whose elements are called coalitions, and $\mu$ is a measure on $\Sigma$. We present a model of awareness according to E. Dekel, B. L. Lipman and A. Rustichini [5]. A different approach of awareness models is discussed in R. Fagin, J. Y. Halpern, Y. Moses and M. Y. Vardi [7].

3.1. Awareness and belief. A belief structure is a tuple $\left\langle\Omega,\left(B_{t}\right)_{t \in N}\right\rangle$ in which $B_{t}: 2^{\Omega} \rightarrow$ $2^{\Omega}$ is trader $t$ 's belief operator. The interpretation of the event $B_{t} E$ is that ' $t$ believes $E$.' An awareness structure is a tuple $\left\langle\Omega,\left(A_{t}\right)_{t \in T},\left(B_{t}\right)_{t \in T}\right\rangle$ in which $\left\langle\Omega,\left(B_{t}\right)_{t \in T}\right\rangle$ is a belief structure and $A_{t}$ is $t$ 's awareness operator on $2^{\Omega}$ defined by

PL

$$
A_{t} E=B_{t} E \cup B_{t}\left(\Omega \backslash B_{t} E\right) \quad \text { for every } E \text { in } 2^{\Omega} .
$$

This is called the plausibility property $\mathbf{P L}$. The interpretation of $A_{t} E$ is that ' $t$ is aware of $E$.' The property $\mathbf{P L}$ says that $t$ is aware of $E$ if he believes it or if he believes that he does not believe it. An event $E$ is said to be $t$ 's evident belief if $T \subseteq B_{t} T$. We can think of it as embodying the essence of what is involved in $t$ making his/her direct observations.

3.2. Associated information structure. We shall give the generalized notion of information partition in the line of Bacharach [3].

Definition 1. The associated information structure $\left(P_{t}\right)_{t \in T}$ with an awareness structure $\left\langle\Omega,\left(A_{t}\right),\left(B_{t}\right)\right\rangle$ is the class of $t$ 's associated information function $P_{t}$ of $\Omega$ into $2^{\Omega}$ defined by $P_{t}(\omega)=\bigcap_{E \in 2^{\Omega}}\left\{E \mid \omega \in E \subseteq B_{t} E\right\}$. (If there is no event $E$ for which $\omega \in E \subseteq B_{t} E$ then we take $P_{t}(\omega)$ to be undefined.) The domain of $P_{t}$ denoted by $\operatorname{Dom}\left(P_{t}\right)$ is the set of all the states at which $P_{t}$ is defined.

The mapping $P_{t}$ is reflexive on its domain in the sense below:

Ref $\omega \in P_{t}(\omega)$ for every $\omega \in \operatorname{Dom}\left(P_{t}\right)$,

and it is said to be transitive if:

$\operatorname{Trn} \quad \xi \in P_{t}(\omega)$ implies $P_{t}(\xi) \subseteq P_{t}(\omega)$ for any $\xi, \omega \in \operatorname{Dom}\left(P_{t}\right)$. 
We call $P_{t}(\omega) t$ 's evidence set at $\omega$, which is interpreted as the basis for all $t$ 's evident beliefs. This is because each $t$ 's evident belief $E$ is decomposed into a union of all evidence sets contained in $E$. Furthermore $P_{t}$ is said to be symmetric if:

Sym $\xi \in P_{t}(\omega)$ implies $P_{t}(\xi) \ni \omega$ for any $\omega$ and $\xi \in \operatorname{Dom}\left(P_{t}\right)$.

REMARK 1. M. Bacharach [3] introduces the strong epistemic model equivalent to the Kripke semantics of the modal logic S5. The model is a tuple $\left\langle\Omega,\left(K_{t}\right)_{t \in T}\right\rangle$, in which $t$ 's knowledge operator $K_{t}: 2^{\Omega} \rightarrow 2^{\Omega}$ satisfies the five postulates: For every $E, F$ of $2^{\Omega}$,
$\mathbf{N} K_{t} \Omega=\Omega$
K $\quad K_{t}(E \cap F)=K_{t} E \cap K_{t} F$;
T $\quad K_{t} F \subseteq F$
$4 K_{t} F \subseteq K_{t} K_{t} F$;
$5 \quad \Omega \backslash K_{t} F \subseteq K_{t}\left(\Omega \backslash K_{t} F\right)$.

t's associated information partition $P_{t}$ with $K_{t}$ is defined by $P_{t}(\omega)=\bigcap_{T \in 2^{\Omega}}\left\{T \in 2^{\Omega} \mid \omega \in\right.$ $\left.K_{t} T\right\}$; it satisfies the postulates Ref, Trn and Sym. This is uniquely determined by $\left(\Omega, K_{t}\right)$. The strong epistemic model can be interpreted as the awareness structure $\left\langle\Omega,\left(A_{t}\right),\left(B_{t}\right)\right\rangle$ such that $B_{t}$ is the knowledge operator. In this situation it is easily verified that $A_{t}$ must be the trivial operator; i.e. $A_{t}(F)=\Omega$ for every $F \in 2^{\Omega}$, and that $\operatorname{Dom}\left(P_{t}\right)=\Omega$.

3.3. Economy with awareness structure. A pure exchange economy under uncertainty is a structure $\mathcal{E}=\left\langle T, \Sigma, \mu, \Omega, \mathbf{e},\left(U_{t}\right)_{t \in T},\left(\pi_{t}\right)_{t \in T}\right\rangle$ consisting of the following structure and interpretations: There are $l$ commodities in each state of the state space $\Omega$; the consumption set of trader $t$ is $\mathbf{R}_{+}^{l} ;(T, \Sigma, \mu)$ is the measure space of the traders; $\mathbf{e}(t, \cdot)$ : $T \times \Omega \rightarrow \mathbf{R}_{+}^{l}$ is $t$ 's initial endowment; $U_{t}: \mathbf{R}_{+}^{l} \times \Omega \rightarrow \mathbf{R}$ is $t$ 's von-Neumann and Morgenstern utility function; $\pi_{t}$ is a subjective prior on $\Omega$ for $t \in T$. For simplicity it is assumed that $\left(\Omega, \pi_{t}\right)$ is a finite probability space with $\pi_{t}$ full support for all $t \in T$; i.e., $\pi_{t}(\omega) \neq 0$ for every $\omega \in \Omega$.

Definition 2. A pure exchange economy with awareness structure is a structure $\mathcal{E}^{A}=$ $\left\langle\mathcal{E},\left(A_{t}\right)_{t \in T},\left(B_{t}\right)_{t \in T},\left(P_{t}\right)_{t \in T}\right\rangle$, in which $\mathcal{E}$ is a pure exchange economy under uncertainty, and $\left\langle\Omega,\left(A_{t}\right)_{t \in T},\left(B_{t}\right)_{t \in T},\left(P_{t}\right)_{t \in T}\right\rangle$ is an awareness structure with $\left(P_{t}\right)_{t \in T}$ the associated information structure. By the domain of the economy $\mathcal{E}^{A}$ we mean $\operatorname{Dom}\left(\mathcal{E}^{A}\right)=$ $\cap_{t \in T} \operatorname{Dom}\left(P_{t}\right)$. The economy is called atomless if $(T, \Sigma, \mu)$ is a non-atomic measure space.

We always assume the condition below:

A-0 $\operatorname{Dom}\left(\mathcal{E}^{A}\right) \neq \emptyset$.

REMARK 2. We can plainly observe that an economy under asymmetric information coincides with an economy $\mathcal{E}^{A}$ with the awareness structure $\left\langle\Omega,\left(A_{t}\right)_{t \in T},\left(B_{t}\right)_{t \in T}\right\rangle$ given by the strong epistemic model, and that $\operatorname{Dom}\left(\mathcal{E}^{A}\right)=\Omega$.

We denote by $\mathcal{F}_{t}$ the field of $\operatorname{Dom}\left(P_{t}\right)$ generated by $\left\{P_{t}(\omega) \mid \omega \in \Omega\right\}$ and denote by $\Pi_{t}(\omega)$ the atom containing $\omega \in \operatorname{Dom}\left(P_{t}\right)$. We denote by $\mathcal{F}$ the join of all $\mathcal{F}_{t}(t \in T)$ on $\operatorname{Dom}\left(\mathcal{E}^{A}\right)$; i.e. $\mathcal{F}=\vee_{t \in T} \mathcal{F}_{t}$, and denote by $\Pi(\omega)$ the atom containing $\omega \in \operatorname{Dom}\left(\mathcal{E}^{A}\right)$ of the field $\mathcal{F}=\vee_{t \in T} \mathcal{F}_{t}$.

We shall often refer to the following conditions: For every $t \in T$,

A-1 $\int_{T} \mathbf{e}(t, \omega) d \mu>0$ for all $\omega \in \Omega$.

A-2 $\mathbf{e}(t, \cdot)$ is $\mathcal{F}_{t}$-measurable on $\operatorname{Dom}\left(P_{t}\right)$; 
A-3 For each $x \in \mathbf{R}_{+}^{l}$, the function $U_{t}(x, \cdot)$ is at least $\mathcal{F}$-measurable on $\operatorname{Dom}\left(\mathcal{E}^{A}\right)$, and the function: $T \times \mathbf{R}_{+}^{l} \rightarrow \mathbf{R},(t, x) \mapsto U_{t}(x, \omega)$ is $\Sigma \times \mathcal{B}$-measurable where $\mathcal{B}$ is the $\sigma$-field of all Borel subsets of $\mathbf{R}_{+}^{l}$.

A-4 For each $\omega \in \Omega$, the function $U_{t}(\cdot, \omega)$ is continuous, strictly increasing and quasiconcave on $\mathbf{R}_{+}^{l}$.

3.4. Expectations. An assignment $\mathbf{x}$ is a mapping from $T \times \Omega$ into $\mathbf{R}_{+}^{l}$ such that for each $\omega \in \Omega$, the function $\mathbf{x}(\cdot, \omega)$ is $\mu$-measurable, and for each $t \in T$, the function $\mathbf{x}(t, \cdot)$ is at least $\mathcal{F}$-measurable on $\operatorname{Dom}\left(\mathcal{E}^{A}\right)$. We denote by $\mathcal{A} s s\left(\mathcal{E}^{A}\right)$ the set of all assignments for the economy $\mathcal{E}^{A}$. By an allocation we mean an assignment a such that for every $\omega \in \Omega$, $\int_{T} \mathbf{a}(t, \omega) d \mu \leq \int_{T} \mathbf{e}(t, \omega) d \mu$. We denote by $\mathcal{A l c}\left(\mathcal{E}^{A}\right)$ the set of all allocations, and for each $t \in T$ we denote by $\mathcal{A l} c\left(\mathcal{E}^{A}\right)_{t}$ the set of all the functions $\mathbf{a}(t, \cdot)$ for $\mathbf{a} \in \mathcal{A} l c\left(\mathcal{E}^{A}\right)$.

We introduce the revised notion of trader's expectation of utility in $\mathcal{E}^{A}$. By $t$ 's exante expectation we mean $\mathbf{E}_{t}\left[U_{t}(\mathbf{x}(t, \cdot)]:=\sum_{\omega \in \operatorname{Dom}\left(P_{t}\right)} U_{t}(\mathbf{x}(t, \omega), \omega) \pi_{t}(\omega)\right.$ for each $\mathbf{x} \in$ $\mathcal{A} s s\left(\mathcal{E}^{A}\right)$. The interim expectation $\mathbf{E}_{t}\left[U_{t}\left(\mathbf{x}(t, \cdot) \mid P_{t}\right]\right.$ is defined by $\mathbf{E}_{t}\left[U_{t}\left(\mathbf{x}(t, \cdot) \mid P_{t}\right](\omega):=\right.$ $\sum_{\xi \in \operatorname{Dom}\left(P_{t}\right)} U_{t}(\mathbf{x}(t, \xi), \xi) \pi_{t}\left(\{\xi\} \cap A_{t}(\{\xi\}) \mid P_{t}(\omega)\right)$.

REMARK 3. It should be noted that we use not the usual notion of posterior $\pi_{t}\left(\{\xi\} \mid P_{t}(\omega)\right)$ but the revised one $\pi_{t}\left(\{\xi\} \cap A_{t}(\{\xi\}) \mid P_{t}(\omega)\right)$. For the discussion about this improvement of the notion of posterior, see Section 4 in Matsuhisa and Usami [12].

\section{The result}

4.1. Ex-post core. An assignment $\mathbf{y}$ is called an ex-post improvement of a coalition $S \in \Sigma$ on an assignment $\mathbf{x}$ at a state $\omega \in \Omega$ if

$\operatorname{Imp1} \mu(S)>0$;

Imp2 $\int_{S} \mathbf{y}(t, \omega) d \mu \leq \int_{S} \mathbf{e}(t, \omega) d \mu ; \quad$ and

Imp3 $U_{t}(\mathbf{y}(t, \omega), \omega)>U_{t}(\mathbf{x}(t, \omega), \omega)$ for almost all $t \in S$.

Definition 3. An allocation $\mathbf{x}$ is said to be an ex-post core allocation of an economy with awareness structure $\mathcal{E}^{A}$ if there is no coalition having an ex-post improvement on $\mathbf{x}$ at any state $\omega \in \operatorname{Dom}\left(\mathcal{E}^{A}\right)$. The ex-post core $\mathcal{C}^{E x P}\left(\mathcal{E}^{A}\right)$ is the set of all the ex-post core allocations of $\mathcal{E}^{A}$.

Let $\mathcal{E}^{A}$ be the economy with awareness structure and $\mathcal{E}^{A}(\omega)$ the economy with complete information $\left\langle T, \Sigma, \mu, \mathbf{e}(\cdot, \omega),\left(U_{t}(\cdot, \omega)\right)_{t \in T}\right\rangle$ for each $\omega \in \Omega$. We denote by $\mathcal{C}\left(\mathcal{E}^{A}(\omega)\right)$ the set of all core allocations for $\mathcal{E}^{A}(\omega)$, and by $\mathcal{W}\left(\mathcal{E}^{A}(\omega)\right)$ the set of all competitive equilibria for $\mathcal{E}^{A}(\omega)$.

Proposition 1. Let $\mathcal{E}^{A}$ be a pure exchange economy with awareness structure satisfying the conditions A-1, A-2 and A-3. Suppose that the economy is atomless. The ex-post core of $\mathcal{E}^{A}$ is non-empty (i.e., $\mathcal{C}^{E x P}\left(\mathcal{E}^{A}\right) \neq \emptyset$ ). Moreover, $\mathcal{C}^{E x P}\left(\mathcal{E}^{A}\right)$ coincides with the set of all assignments $\mathbf{x}$ such that $\mathbf{x}(\cdot, \omega)$ is a core allocation for the economy $\mathcal{E}^{A}(\omega)$ for all $\omega \in \operatorname{Dom}\left(\mathcal{E}^{A}\right):$ i.e.,

$$
\left.\mathcal{C}^{E x P}\left(\mathcal{E}^{A}\right)=\left\{\mathbf{x} \in \mathcal{A} l c\left(\mathcal{E}^{A}\right) \mid \mathbf{x}(\cdot, \omega)\right) \in \mathcal{C}\left(\mathcal{E}^{A}(\omega)\right) \text { for all } \omega \in \operatorname{Dom}\left(\mathcal{E}^{A}\right)\right\} .
$$

Proof. Modify the proof of Theorem 3.1 in Einy et al [6]. 
4.2. Expectations equilibrium in awareness. A price system is a non-zero function $p$ : $\Omega \rightarrow \mathbf{R}_{+}^{l}$ which is $\mathcal{F}$-measurable on $\operatorname{Dom}\left(\mathcal{E}^{A}\right)$. We denote by $\Delta(p)$ the partition on $\Omega$ induced by $p$, and denote by $\sigma(p)$ the field of $\Omega$ generated by $\Delta(p)$. The budget set of a trader $t$ at a state $\omega$ for a price system $p$ is defined by $B_{t}(\omega, p):=\left\{x \in \mathbf{R}_{+}^{l} \mid p(\omega) \cdot x \leq\right.$ $p(\omega) \cdot \mathbf{e}(t, \omega)\}$. Define the mapping $\Delta(p) \cap P_{t}: \operatorname{Dom}\left(P_{t}\right) \rightarrow 2^{\Omega}$ by $\left(\Delta(p) \cap P_{t}\right)(\omega):=$ $\Delta(p)(\omega) \cap P_{t}(\omega)$; it is plainly observed that $\Delta(p) \cap P_{t}$ satisfies Ref. We denote by $\sigma(p) \vee \mathcal{F}_{t}$ the smallest $\sigma$-field containing both the fields $\sigma(p)$ and $\mathcal{F}_{t}$.

Definition 4. An expectations equilibrium in awareness for an economy $\mathcal{E}^{A}$ with awareness structure is a pair $(p, \mathbf{x})$, in which $p$ is a price system and $\mathbf{x}$ is an assignment satisfying the following conditions:

EA1 $\mathbf{x}$ is an allocation;

EA2 For almost all $t \in T$ and for every $\omega \in \Omega, \mathbf{x}(t, \omega) \in B_{t}(\omega, p)$;

EA3 For almost all $t \in T$, if $\mathbf{y}(t, \cdot): \Omega \rightarrow \mathbf{R}_{+}^{l}$ is $\mathcal{F}$-measurable on $\operatorname{Dom}\left(\mathcal{E}^{A}\right)$ with $\mathbf{y}(t, \omega) \in B_{t}(\omega, p)$ for all $\omega \in \Omega$, then $\mathbf{E}_{t}\left[U_{t}(\mathbf{x}(t, \cdot)) \mid \Delta(p) \cap P_{t}\right](\omega) \geq \mathbf{E}_{t}\left[U_{t}(\mathbf{y}(t, \cdot)) \mid \Delta(p) \cap\right.$ $\left.P_{t}\right](\omega)$ pointwise on $\operatorname{Dom}\left(P_{t}\right)$;

EA4 For every $\omega \in \operatorname{Dom}\left(\mathcal{E}^{A}\right), \int_{t \in T} \mathbf{x}(t, \omega) d \mu=\int_{t \in T} \mathbf{e}(t, \omega) d \mu$.

The allocation $\mathbf{x}$ in $\mathcal{E}^{A}$ is called an expectations equilibrium allocation in awareness for $\mathcal{E}^{A}$.

We denote by $E A\left(\mathcal{E}^{A}\right)$ the set of all the expectations equilibria of a pure exchange economy $\mathcal{E}^{A}$, and denote by $\mathcal{A}\left(\mathcal{E}^{A}\right)$ the set of all the expectations equilibrium allocations in awareness for the economy.

THEOREM 2. Let $\mathcal{E}^{A}$ be a pure exchange economy with awareness structure satisfying the conditions A-1, A-2, A-3 and A-4. Suppose that the economy is atomless. Then there exists an expectations equilibrium in awareness for the economy; i.e., $E A\left(\mathcal{E}^{A}\right) \neq \emptyset$.

Before proceeding with the proof we should note that:

LEMMA 3. Let $\mathcal{E}^{A}$ be a pure exchange economy with awareness structure satisfying the condition A-3. For every $t \in T$ and for every $\omega \in \operatorname{Dom}\left(\mathcal{E}^{A}\right)$, the event $\left(\Delta(p) \cap P_{t}\right)(\omega)$ can be decomposed into the disjoint union of all the components $\Pi(\xi)$ for $\xi \in\left(\Delta(p) \cap P_{t}\right)(\omega)$; i.e., there exist $\Pi\left(\xi_{1}\right), \ldots, \Pi\left(\xi_{m}\right) \in \mathcal{F}$ such that $\left(\Delta(p) \cap P_{t}\right)(\omega)=\cup_{k=1}^{m} \Pi\left(\xi_{k}\right)$ as disjoint union. Moreover, if $\mathbf{x}$ is an assignment for $\mathcal{E}^{A}$ then we obtain that

$$
\mathbf{E}_{t}\left[U_{t}(\mathbf{x}(t, \cdot)) \mid \Delta(p) \cap P_{t}\right](\omega)=\sum_{k=1}^{m} \frac{\pi_{t}\left(\Pi\left(\xi_{k}\right)\right)}{\pi_{t}\left(\left(\Delta(p) \cap P_{t}\right)(\omega)\right)} U_{t}\left(\mathbf{x}\left(t, \xi_{k}\right), \xi_{k}\right),
$$

where the left hand side is independent of the choices of the representatives $\left\{\xi_{1}, \xi_{2}, \ldots \xi_{m}\right\}$ in the partition $\left\{\Pi\left(\xi_{k}\right) \mid 1 \leq k \leq m\right\}$ of $\left(\Delta(p) \cap P_{t}\right)(\omega)$.

Proof of Theorem 2. In view of the existence theorem of a competitive equilibrium for an atomless economy with complete information (cf. Theorem 9 in Debreu [4]), it follows that there exists a $\left(p^{*}(\omega), \mathbf{x}^{*}(\cdot, \omega)\right) \in \mathcal{W}\left(\mathcal{E}^{A}(\omega)\right)$ for each $\omega \in \Omega$ by the conditions A-1,A-2, A-3 and A-4. Define the pair $(p, \mathbf{x})$ as follows: For each $\omega \in \Omega$, denote $\mathbf{x}(t, \xi):=\mathbf{x}^{*}(t, \omega)$ for all $\xi \in \Pi(\omega)$ and $\omega \in \operatorname{Dom}\left(\mathcal{E}^{A}\right)$, and set $p(\xi):=p^{*}(\omega)$ for all $\xi \in$ $\Pi(\omega)$ and $\omega \in \operatorname{Dom}\left(\mathcal{E}^{A}\right), p(\xi):=p^{*}(\omega)$ for $\omega \notin \operatorname{Dom}\left(\mathcal{E}^{A}\right)$. Then we can verify that 
$(p, \mathbf{x})$ is an expectations equilibrium in awareness for $\mathcal{E}^{A}$ : For EA3. On noting that $\mathcal{E}^{A}(\xi)=\mathcal{E}^{A}(\omega)$ for any $\xi \in \Pi(\omega)$, it follows that $(p(\xi), \mathbf{x}(t, \xi)) \in \mathcal{W}\left(\mathcal{E}^{A}(\omega)\right)$ for every $\omega \in \Omega$, and thus we can observe EA3 by Eq (1). The other conditions in Definition 4 are easily verified.

REMARK 4. As for the notion of expected equilibrium in awareness, we impose the condition that the assignment is $\mathcal{F}$-measurable under the budget constraints in EA3. However, in the usual notion of rational expectations equilibrium for an economy under asymmetric information, each rational expectations equilibrium allocation maximizes trader $t$ 's interim expectations on all of the $\sigma(p) \vee F_{t}$-measurable assignments under $t$ 's budget constraint (cf. Einy et al. [6]). It seems problematic that a trader might not maximize their expected utilities on the assignments under the finest common information.

4.3. Proof of the main theorem. We can now state explicitly the main theorem:

THEOREM 4. Let $\mathcal{E}^{A}$ be a pure exchange economy with awareness structure satisfying the conditions $\mathbf{A - 1}, \mathbf{A - 2}, \mathbf{A - 3}$ and $\mathbf{A - 4}$. Suppose that the economy is atomless. Then the ex-post core coincides with the set of all expectations equilibrium allocations in awareness; i.e., $\mathcal{C}^{\operatorname{ExP}}\left(\mathcal{E}^{A}\right)=\mathcal{A}\left(\mathcal{E}^{A}\right)$.

Because $\mathcal{E}^{A}(\omega)$ is an atomless economy for each $\omega \in \Omega$, we can observe that $\mathcal{C}\left(\mathcal{E}^{A}(\omega)\right)$ $=\mathcal{W}\left(\mathcal{E}^{A}(\omega)\right)$ for any $\omega \in \Omega$ by the core equivalence theorem of Aumann ([1], [2]). The main theorem immediately follows from Proposition 1 together with Proposition 5 as below.

Proposition 5. Let $\mathcal{E}^{A}$ be an economy with awareness structure satisfying the conditions A-1, A-2, A-3 and A-4. Then the set of all expectations equilibrium allocations in awareness $\mathcal{A}\left(\mathcal{E}^{A}\right)$ coincides with the set of all the assignments $\mathbf{x}$ such that $\mathbf{x}(\cdot, \omega)$ is a competitive equilibrium allocation for the economy with complete information $\mathcal{E}^{A}(\omega)$ for all $\omega \in \operatorname{Dom}\left(\mathcal{E}^{A}\right)$; i.e.,

$$
\begin{aligned}
& \mathcal{A}\left(\mathcal{E}^{A}\right)=\left\{\mathbf{x} \in \mathcal{A l c}\left(\mathcal{E}^{A}\right) \mid \text { There is a price system } p\right. \text { such that } \\
& \left.\qquad(p(\omega), \mathbf{x}(\cdot, \omega)) \in \mathcal{W}\left(\mathcal{E}^{A}(\omega)\right) \text { for all } \omega \in \operatorname{Dom}\left(\mathcal{E}^{A}\right)\right\} .
\end{aligned}
$$

Proof of Theorem 4. Let $\mathbf{x} \in \mathcal{A}\left(\mathcal{E}^{A}\right)$. By Proposition $5,(p(\omega), \mathbf{x}(\cdot, \omega)) \in \mathcal{W}\left(\mathcal{E}^{A}(\omega)\right)$ for each $\omega \in \operatorname{Dom}\left(\mathcal{E}^{A}\right)$, and so $\left.\mathbf{x}(\cdot, \omega)\right) \in \mathcal{C}\left(\mathcal{E}^{A}(\omega)\right)$ by the theorem of Aumann ([1], [2]). By Proposition 1, $\mathcal{C}^{E x P}\left(\mathcal{E}^{A}\right) \supseteq \mathcal{A}\left(\mathcal{E}^{A}\right)$. The converse shall be shown as follows: Let $\mathbf{x} \in \mathcal{C}^{E x P}\left(\mathcal{E}^{A}\right)$. By Proposition $1, \mathbf{x}(\cdot, \omega) \in \mathcal{C}\left(\mathcal{E}^{A}(\omega)\right)$ for every $\omega \in \operatorname{Dom}\left(\mathcal{E}^{A}\right)$. By the above theorem of Aumann, there is $p^{*}(\omega) \in \mathbf{R}_{+}^{l}$ such that $\left(p^{*}(\omega), \mathbf{x}(\cdot, \omega)\right) \in \mathcal{W}\left(\mathcal{E}^{A}(\omega)\right)$. Defining the price system $p$ by the same way in the proof of Theorem 2, we can obtain that $(p(\omega), \mathbf{x}(\cdot, \omega)) \in \mathcal{W}\left(\mathcal{E}^{A}(\omega)\right)$ for every $\omega \in \operatorname{Dom}\left(\mathcal{E}^{A}\right)$. By Proposition $5, \mathcal{C}^{E x P}\left(\mathcal{E}^{A}\right) \subseteq$ $\mathcal{A}\left(\mathcal{E}^{A}\right)$.

Proof of Proposition 5. Let $\mathbf{x} \in \mathcal{A}\left(\mathcal{E}^{A}\right)$ and $(p, \mathbf{x}) \in E A\left(\mathcal{E}^{A}\right)$. We shall show that $(p(\omega), \mathbf{x}(\cdot, \omega)) \in \mathcal{W}\left(\mathcal{E}^{A}(\omega)\right)$ for any $\omega \in \Omega$ : Suppose to the contrary that there exist a state $\omega_{0} \in \operatorname{Dom}\left(\mathcal{E}^{A}\right)$ and non-null set $S \subseteq T$ with the property: For each $s \in S$ there is an $\mathbf{a}\left(s, \omega_{0}\right) \in B_{s}\left(\omega_{0}, p\right)$ such that $U_{s}\left(\mathbf{a}\left(s, \omega_{0}\right), \omega_{0}\right)>U_{s}\left(\mathbf{x}\left(s, \omega_{0}\right), \omega_{0}\right)$. Define the function $\mathbf{y}: T \times \Omega \rightarrow \mathbf{R}_{+}^{l}$ by: $\mathbf{y}(t, \xi):=\mathbf{a}\left(t, \omega_{0}\right)$ for $\xi \in \Pi\left(\omega_{0}\right), \mathbf{y}(t, \xi):=\mathbf{x}(t, \xi)$ otherwise. On 
noting that $\mathbf{y}(t, \cdot)$ is $\mathcal{F}$-measurable on $\operatorname{Dom}\left(\mathcal{E}^{A}\right)$ and $\pi_{t}$ is full support, we can obtain by Eq (1) that for all $s \in S, \mathbf{E}_{s}\left[U_{s}(\mathbf{x}(s, \cdot)) \mid \Delta(p) \cap P_{s}\right]\left(\omega_{0}\right)<\mathbf{E}_{s}\left[U_{s}(\mathbf{y}(s, \cdot)) \mid \Delta(p) \cap P_{s}\right]\left(\omega_{0}\right)$, contrary to $(p, \mathbf{x}) \in E A\left(\mathcal{E}^{A}\right)$. The converse will be shown as follows: Let $\mathbf{x} \in \mathcal{A} s s\left(\mathcal{E}^{A}\right)$ with $(p(\omega), \mathbf{x}(\cdot, \omega)) \in \mathcal{W}\left(\mathcal{E}^{A}(\omega)\right)$ for all $\omega \in \operatorname{Dom}\left(\mathcal{E}^{A}\right)$. Define the price system $p^{*}: \Omega \rightarrow \mathbf{R}_{+}^{l}$ by $p^{*}(\xi):=p(\omega)$ for all $\xi \in \Pi(\omega)$ and $\omega \in \operatorname{Dom}\left(\mathcal{E}^{A}\right), p^{*}(\xi):=p(\omega)$ for $\omega \notin \operatorname{Dom}\left(\mathcal{E}^{A}\right)$. We can observe that $\left(p^{*}, \mathbf{x}\right) \in E A\left(\mathcal{E}^{A}\right)$ : For EA3. Let $\mathbf{y}(t, \cdot): \Omega \rightarrow \mathbf{R}_{+}^{l}$ be an $\mathcal{F}$-measurable function with $\mathbf{y}(t, \omega) \in B_{t}\left(\omega, p^{*}\right)$ for all $\omega \in \operatorname{Dom}\left(\mathcal{E}^{A}\right)$. Since $\left(p^{*}(\omega), \mathbf{x}(\cdot, \omega)\right) \in \mathcal{W}\left(\mathcal{E}^{A}(\omega)\right)$ it follows that $U_{t}(\mathbf{x}(t, \omega), \omega) \geq U_{t}(\mathbf{y}(t, \omega), \omega)$ for almost all $t \in T$. Therefore by Eq (1), $\mathbf{E}_{t}\left[U_{t}(\mathbf{x}(t, \cdot)) \mid \Delta\left(p^{*}\right) \cap P_{t}\right](\omega) \geq \mathbf{E}_{t}\left[U_{t}(\mathbf{y}(t, \cdot)) \mid \Delta\left(p^{*}\right) \cap P_{t}\right](\omega)$, as required. The other conditions in Definition 4 can be easily verified.

5. Concluding remarks. Our real concern in this article is about relationship between players' beliefs and their decision making, especially when and how the players take corporate actions under their decisions. We focus on extending the core equivalence theorem of Aumann ([1], [2]) to an economy with traders having 'awareness and belief' model. We have shown that the nature of the equivalence between core and expected equilibrium allocations is dependent not on common-belief assumption nor on the partition structure of traders' information, but on the structure of awareness and belief when each player receives information. It is appropriate to comment on the assumptions in our results. The suppression of any of A-1 to A-4 renders Theorem 2 vulnerable to the discussion and the example proposed in Remarks 4.6 of Matsuhisa and Ishikawa [10].

\section{References}

[1] R. J. Aumann, Markets with a continuum of traders, Econometrica 32 (1964), 39-50.

[2] R. J. Aumann, Existence of competitive equilibrium in markets with a continuum of traders, Econometrica 34 (1966), 1-17.

[3] M. O. Bacharach, Some extensions of a claim of Aumann in an axiomatic model of knowledge, Journal of Economic Theory 37 (1985), 167-190.

[4] G. Debreu, Existence of competitive equilibrium, in: Handbook of Mathematical Economics, Volume 2, K. J. Arrow and M. D. Intriligator (eds), North-Holland, Amsterdam, 1982, 697-744,

[5] E. Dekel, B.L. Lipman, and A. Rustichini, Standard state-space models preclude unawareness, Econometrica 66 (1998), 159-173.

[6] E. Einy, D. Moreno, and B. Shitovitz, Rational expectations equilibria and the ex-post core of an economy with asymmetric information, Journal of Mathematical Economics 34 (2000), 527-535.

[7] R. Fagin, J. Y. Halpern, Y. Moses and M. Y. Vardi, Reasoning about Knowledge. The MIT Press, Cambridge, Massachusetts, London, England, 1995.

[8] J. Geanakoplos, Game theory without partitions, and applications to speculation and consensus, Cowles Foundation Discussion Paper No. 914, 1989. (Available at http://cowles.econ.yale.edu)

[9] F. Forges, E. Minelli, and R. Vohra, Incentive and core of an exchange economy: a survey, Journal of Mathematical Economics 38 (2002), 1-41. 
[10] T. Matsuhisa and R. Ishikawa, Rational expectations can preclude trades, Working paper. Hitotsubashi Discussion Paper Series No. 2002-1, 2002. (Available at http://wakame.econ.hit-u.ac.jp/ koho/english/3research/3index.html).

[11] T. Matsuhisa, R. Ishikawa and Y. Hoshino, Core equivalence in economy under generalized information, Working paper. Hitotsubashi Discussion Paper Series No. 2002-12, 2002. (Available at http://wakame.econ.hit-u.ac.jp/)

[12] T. Matsuhisa and S.-S. Usami, Awareness, belief and agreeing to disagree, Far East Journal of Mathematical Sciences 2 (2000), 833-844.

[13] O. Volij, Communication, credible improvements and the core of an economy with asymmetric information, International Journal of Game Theory 29 (2000), 63-79.

[14] R. Wilson, Information, efficiency, and the core of an economy, Econometrica 40 (1978), 807-816. 